\title{
Regenerative Engineering in Maxillofacial Reconstruction
}

\author{
Jonathan L. Shaul ${ }^{1}$ • Betsy K. Davis ${ }^{1,2}$ • Karen J. L. Burg ${ }^{1,3}$
}

Received: 26 July 2015 / Accepted: 25 January 2016 / Published online: 23 June 2016

(C) The Regenerative Engineering Society 2016

\begin{abstract}
Oral cancer is the sixth most common cancer worldwide, with approximately 275,000 new cases each year. In some countries, mortality rates reach as high as $70 \%$. For patients that survive, bodily functions of speaking, swallowing, and chewing are severely compromised. Although there has been improvement in free tissue transfer techniques and virtual planning with implant placement, maxillofacial reconstruction techniques need refinement to allow greater improvement in functional outcomes and quality of life. Regenerative engineering principles provide a potential means of improving maxillofacial tissue regeneration. Maxillofacial reconstruction requires unique insights to maximize clinical impact. Because traditional cancer treatment can include radiation therapy, defect sites may experience hypoxia and experience thrombosis and fibrosis, which complicate restoration [1]. Several cell sources, such as periosteal-derived progenitor cells (PDPCs) and bone marrow-derived mesenchymal stem cells (BMSCs) have been evaluated for use in maxillofacial reconstruction, with variable success. From our comprehensive review of the literature, there is a significant need to further the application
\end{abstract}

Karen J. L. Burg

kburg@uga.edu

1 Department of Bioengineering and Institute for Biological Interfaces of Engineering, Clemson University, 401 Rhodes Engineering Research Center, Clemson 29634, USA

2 Department of Otolaryngology, Medical University of South Carolina, MUSC Rutledge Tower, Charleston 29464, USA

3 Department of Small Animal Medicine and Surgery, College of Veterinary Medicine, University of Georgia, 501 D.W. Brooks Drive, Athens, GA 30602, USA of regenerative engineering principles to maxillofacial regeneration following oral cancer treatment.

\section{Lay Summary}

The prevalence of oral cancer and limitations of treatments invites regenerative engineering approaches. While combatting cancer, tissue removal, radiation, and/or chemotherapy often significantly reduce the patient's quality of life. A high-quality "functional outcome" following treatment means the ability to readily perform the seemingly simplest of biological tasks that humans often take for granted-swallowing, breathing, coughing, and speaking intelligibly. Indeed, damage due to clinical treatments may include normal tissues at the cancer site but can also extend to surrounding tissues such as teeth. A regenerative engineering approach, involving tissue enhancement with cellular materials, would allow tissue to be rebuilt following and during potentially harmful clinical treatments.

Keywords Bone $\cdot$ Cell Biology $\cdot$ Oral Cancer $\cdot$ Radiation Tissue Engineering

\section{Background and Significance}

In the USA, there are approximately 40,250 new diagnoses of oral cancer annually. Of these, approximately 8000 patients will succumb to the disease each year [2]. Oral cancer is the sixth most common cancer in the world, with approximately 275,000 new cases annually [3]. In countries such as India and Brazil, mortality rates can reach as high as $70 \%$ [4] per year. Typically, the incidence of oral cancer is related to behavioral and lifestyle factors, which can be potentially minimized with changes to activities of daily living. Specifically, tobacco and alcohol usage have been linked with increased incidence of oral cancer. In countries such as India, consumption of pan 
(tobacco mixed with betel leaves and lime) is thought to be a major cause for the abnormally high rates of oral cancer [4]. Furthermore, incidence rates indicate a higher rate of oral cancer in males. Current theories are that elevated oral cancer rates are caused by increased consumption of alcohol and tobacco by males.

In the majority of new diagnoses, patients are over the age of 50 and have prolonged exposure to risk factors. However, infection of the human papillomavirus (HPV) has been shown to cause oral cancer, which tends to afflict a younger population $[4,5,6,7,8]$. HPV-positive oropharyngeal cancers tend to have a higher survivability rate than HPV-negative cancers. However, the rates of HPV-positive cancers are rapidly increasing, while HPV-negative oropharyngeal cancers are decreasing with the decrease in smoking in the USA. In the USA, a similar observation can be made, with the rate of HPV-positive oropharyngeal cancers increasing at a rate of $225 \%$ from 1988 to 2004. Current projections predict HPVpositive oropharyngeal cancers surpassing the number of HPV-positive cervical cancers by 2020 [9]. Similarly, in Sweden, oral cancer caused by HPV has dramatically increased from $23 \%$ in the 1970 s to $97 \%$ in 2007 . In recent years, the development of HPV vaccinations has caused health care professionals to recommend the vaccinations of both genders, in order to attempt to stem the tide of future cancer diagnoses [9].

\section{Clinical Relevance}

\section{Current Clinical Approaches}

\section{Clinical Treatments}

Currently, treatment methodologies for patients that suffer from oral cancer are confined to surgical resection/reconstruction, radiation therapy, and/or chemotherapy. These methods result in varying functional outcomes. Typically, higher-stage tumors cause a more significant decline in functional outcome. Resection of the tumor leaves a defect that can negatively impact the quality of life for the patients. Inability to swallow, difficulty breathing, and inability to speak intelligibly negatively affect the quality of life [10]. Ideally, the defect can be closed primarily and, if successful, patients have a significant improvement in quality of life [11]. However, radiotherapy and chemotherapy tend to impact the quality of life. Radiotherapy and chemotherapy tend to decrease speech and swallowing over the first 6 months of treatment. Some of these impacts can be improved by the 1-year postoperative time point. However, the quality of life is impacted through the entire recovery time [12].

Prior to therapy, a dental evaluation is performed to ensure that the teeth of the patient are healthy enough to undergo radiation therapy. Because radiation can impair healthy vascularization, dental infections are unable to heal following radiation therapy. Nonviable teeth must be removed and all dental prophylactic procedures must be performed prior to therapy [13]. Radiation therapy, in a dose-dependent manner, can significantly influence the surrounding tissue. Specifically, mucus membranes can become inflamed and cause the influx of inflammatory cytokines, which can dramatically alter the microenvironment. Radiation treatment also causes deoxyribonucleic acid (DNA) damage and causes the formation of reactive oxygen metabolites. Both of these factors result in hypoplasia [1]. Furthermore, higher doses of radiation can cause significant damage to the remaining healthy bone by killing the endothelial cells of the vasculature and damaging the endosteum and periosteum [13, 14]. Therefore, the same factors that limit tumor formation limit regeneration of healthy tissue. Furthermore, endothelial cells play a significant role in the angiogenesis of damaged tissue; the decrease in endothelial cells limits integration of transplanted tissues [15]. In order to minimize the risk to surrounding tissue, intensity-modulated radiotherapy (IMRT) has been implemented. IMRT radiation dose is varied based on the density of the cancerous region. Through computer modeling and beam modifiers, dosages can be targeted to specific regions, minimizing the dosage to surrounding sensitive tissues [16]. Specifically, the implementation of IMRT has helped to reduce the risk of osteonecrosis and spare the salivary glands of patients $[17,18]$. If significant progression of the tumor is detected, chemotherapy may be performed in conjunction with radiotherapy. While initially thought to be tissue and organ-sparing, radiotherapy may be correlated to chronic fibrosis, edema, and some organ dysfunction. Similarly, chemotherapy has shown systemic organ damage, specifically nervous tissue, and organ fibrosis [19].

Maxillary defects are usually reconstructed surgically with a free tissue transfer or with a prosthetic with an obturator. Both surgical and prosthetic rehabilitation have limitations. With surgical resection of the cancerous mass, the vascularity in the site may be compromised. Postoperative radiation therapy also reduces the vascularity, potentially leading to infection and improper healing of tissue surrounding dental implants [20, 21]. In addition to those risks, patients experience difficulty breathing, swallowing, eating, and speaking. This loss of function can lead to depression among patients [10]. Furthermore, patients will suffer from destruction or removal of glandular structures during surgery or destruction during radiotherapy, which will impair salivary gland function and therefore reduce the ability of patients to eat properly $[1,13]$. Finally, maxillectomies can result in facial disfiguration, which often leads to decreases in patient morale and mental state. Due to these limitations, closure of the defect site is of primary concern as a means to improve patient function and quality of life [22]. 
Overall, obturators do an adequate job of closing the defect site in oral cancer patients, but there are significant limitations that must be addressed to provide patients with the highest quality of function. Specifically, obturator fabrication and maintenance are very labor and time intensive, and obturators are incapable of replacing native biologic qualities. Due to radiation side effects, patients with obturators experience hypo-salivary production for the remainder of their lives, which adversely affects swallowing [1, 10, 23, 24]. Furthermore, the patients must properly care for their obturators by removing and cleaning them regularly and maintaining a regular check-up schedule with the prosthodontist.

For a large number of patients, autologous free flap transfers significantly improve maxillary defects. When properly performed, the defect site is sealed with autologous tissue to prevent transfer of food and fluids from the oral to the nasal cavities [25]. However, this procedure can have significant complications. Additional tissue can add too much bulk and prevent dental implant placement. Furthermore, the excess tissue can cause breathing and communication difficulties (Fig. 1) [26]. Additionally, diet for patients may be permanently altered toward a softer diet [25, 27]. Free flap transfers require a second surgical site that provides an increased risk of morbidity and mortality, such as lameness and infection [25]. Autologous grafts provide advantages over synthetic prostheses in that the necessity for longterm patience compliance is lowered. However, the autologous grafts require multiple surgical procedures and have the potential for significant complications as well as variable functional results. Furthermore, grafts do not allow direct visualization of the tumor site for postoperative cancer recurrence evaluations as compared with synthetic obturators, which can be removed to assess the defect site.

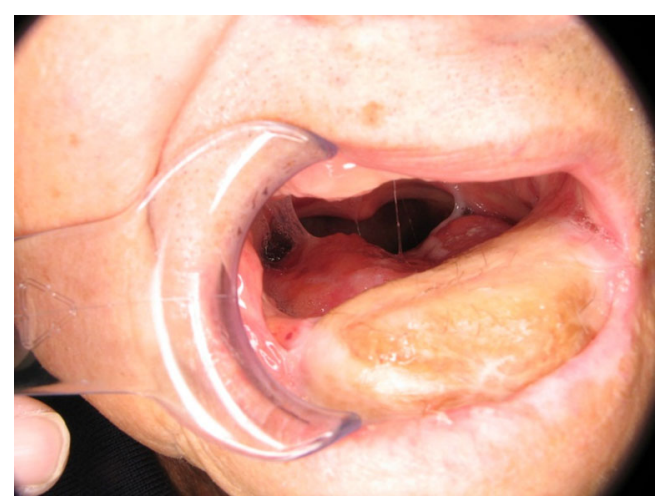

Fig. 1 Patient who underwent free flap transfer to recreate a defect in the maxillary, but excessive bulk prevented dental implants. Photo was taken and used with patient permission to the Medical University of South Carolina
Function and Patient Quality of Life following Cancer Treatment

Patients suffer from significant issues that can lead to additional psychological issues such as depression and anxiety $[10,28]$. The loss of bodily functions of speaking, swallowing, and chewing strongly correlates to patient depression and anxiety following reconstruction. These patients experience loss of function, which limits both recreational and occupational activities. The majority of patients experience moderate to severe pain on a daily basis, which can limit function. Difficulties with chewing, swallowing, and speaking are strongly correlated to rates of strong depression and feelings of hopelessness, which have significant negative impact on a patient's mental and physical health [29]. With improvement in reconstruction, psychological issues have declined to approximately $25 \%$ of patients who undergo these procedures [29]. For patients that undergo maxillary reconstruction, the treatment method has a significant impact on the quality of life for the patient. For patients who have obturators, mastication, speech intelligibility, and comfort in speaking to others are significantly decreased [30]. Free flap transfers have slightly improved functional outcomes when compared with functional outcomes of obturators, but functional outcomes of free flaps are much less effective as compared with outcomes of control patients who have not undergone treatment for oral cancer [30]. Future work must address restoration of function, as it is critical for both the physical and mental health of the patient.

\section{Regenerative Engineering Approaches}

Bone regenerative engineering approaches have been applied to maxillofacial reconstruction. Traditionally, tissue engineering uses a three-part implant consisting of a cellular source, scaffold material, and exogenous growth factors. When compared with other tissue engineering applications, maxillary tissue engineering has unique situations that warrant considerations in all three parts. In addition to mechanical requirements of maxillary tissue, there are significant physiological components of the maxilla that need recreation by a tissue engineering solution. These functionalities include glands, vasculature to the teeth, and attachment for musculature. Because of the location of the maxilla, a tissue-engineered construct would be directly exposed to the oral and nasal cavities, allowing visualization of the site, but increasing exposure to viral and bacterial factors. The compromised vascularity to the site must be considered when planning a regeneration strategy; additionally, the effects of localized cancer treatment must be factored into reconstructive techniques. A variety of progenitor cell types have been investigated, including adipose stem cells (Fig. 2), bone marrow-derived mesenchymal stem cells (BMSCs), and, in limited use, periostealderived progenitor cells (PDPCs). 
Fig. 2 a Preoperative defect site. b Twenty-eight-month follow-up following reconstruction reveals ectopic bone formed from adipose-derived stem cells. Reprinted from [96], with permission from Elsevier
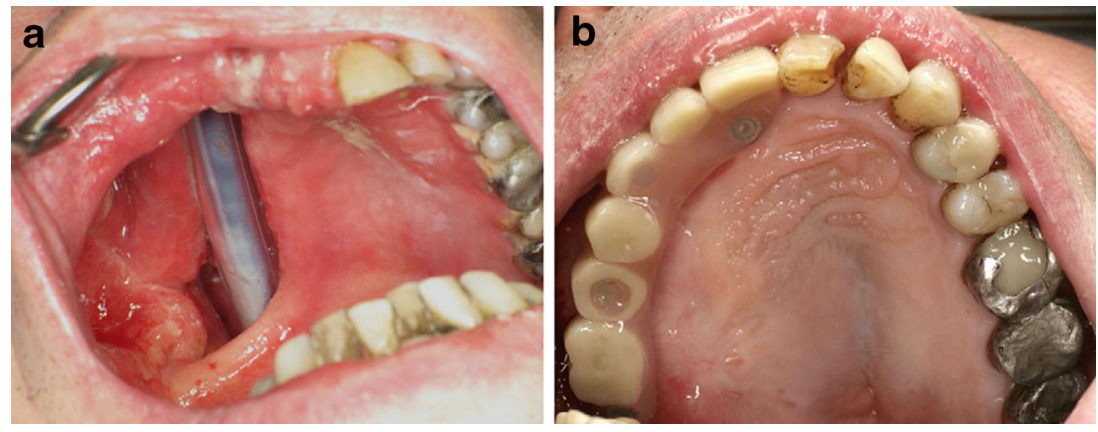

\section{Cellular Sources}

\section{Bone Marrow-Derived Mesenchymal Stem Cells Bone} marrow is a complex tissue that consists of a large variety of cellular components as well as a supporting matrix, termed stroma. Hematopoiesis, formation of blood components from stem cells, is considered the main function. However, bone marrow also contains stromal cells which include mesenchymal stem cells that are capable of differentiating into tissues such as bone, adipose, and cartilage [31, 32].

Cells designated for use in regenerative engineering are isolated via aspirate, typically from the iliac crest, and expanded in vitro. In order to correctly obtain cells with multi-lineage potential, cells must be grown in culture to produce colonyforming units and, subsequently, passaged [33, 34, 35]. More recently, with the use of fluorescence-activated cell sorting (FACS), mesenchymal stem cells can be separated from other cell types via identification of surface markers. Jones and coworkers demonstrated this concept by fluorescently labeling markers such as cluster of differentiation (CD) 45 , which is a leukocyte marker found on mesenchymal stem cells [36]. Once expanded, differentiation can occur through a variety of methodologies, including chemical signals, growth factors, and mechanical conditions. Furthermore, Zhou and coworkers demonstrated that BMSCs migrate preferentially to defect sites. This migration was detected using green fluorescent protein (GFP)-expressing bone marrow mesenchymal stem cells [37]. The cells were isolated and characterized using immunohistochemistry to evaluate expression of CD14, CD34, CD45, CD90, CD105, and CD106, which are commonly expressed by mesenchymal stem cells $[37,38]$.

\section{Bone Marrow Mesenchymal Stem Cells Used in Tissue} Engineering Due to the proliferative potential of mesenchymal stem cells, BMSCs have been evaluated for use in tissue regeneration of the maxilla following oral cancer treatment. Many research groups focus on the use of BMSCs for maxillary reconstruction in an attempt to mimic or expedite the regeneration process. Many of the same inflammatory mediators that are secreted by wounds are found in the tumor microenvironment and are purported to be involved in attracting MSCs to these sites [39, 40, 41, 42].

Similarly, BMSCs have shown in vitro and in vivo success in forming bone tissue [43]. In one study, BMSCs were collected from maxillofacial tissue during maxillofacial treatment. The cells were cultured and characterized using FACS for cell marker expression. BMSCs were differentiated in vitro and analyzed for alkaline phosphatase, Oil-Red-O, and Alcian blue for osteogenic, adipogenic, and chondrogenic potential, respectively, using histological techniques. Additionally, cells were genetically engineered with bone morphogenetic protein-2 (BMP-2), which can induce bone formation. Following gene delivery, these cells were injected into the thighs of mice for a period of 3 weeks for ectopic growth. Finally, these cells were implanted into mandibular defects; the defects showed signs of bone regeneration after a period of 8 weeks. While this study demonstrated $84 \%$, by size, bone regeneration of a defect within the mandible, the study required genetic modification of the cells to produce endogenous BMP-2; additionally, the treatment required significant in vitro procedures, which lengthens the recovery time for patients undergoing the treatment [43]. However, this study demonstrated the potential impact of BMPs on defect regeneration in the maxillofacial region. Furthermore, BMSCs obtained from the maxillofacial region demonstrated potential for bone tissue regeneration, which in turn shows the potential for minimizing simultaneous surgical procedures at multiple sites.

In an attempt to recreate flat bone of the skull, Lucaciu and coworkers isolated BMSCs from mice femurs and combined them in vitro with deciduous red deer antler to attempt to stimulate bone formation [44]. Deer antler was chosen as a scaffold due to high levels of polypeptides such as insulin-like growth factor-1 (IGF-1), IGF-2, BMP-2, and BMP-4. The group theorized that growth of in vitro bone would provide clinicians a viable alternative to traditional graft materials. After harvesting BMSCs, the cells were cultured and expanded. The cells were then seeded onto the deer antler scaffolds and the composites were then exposed to basal osteogenic medium with supplements of $\beta$-glycerophosphate, ascorbic acid, and insulin. Additionally, separate samples were exposed to complex osteogenic medium, which included the additives 
and the presence of growth factors such as BMP-2 and transforming growth factor- $\beta$ (TGF- $\beta$ ). In order to produce bone tissue, the composites incubated for a period of 30 days. The engineered tissue was then implanted into defects within the parietal bone of the mouse skull. In the mouse model, the construct successfully repaired the defect. Improved bone regeneration was shown in composites cultured in complex differentiation medium. Throughout the composite construction, the researchers used in vitro techniques to engineer an artificial bone for reconstruction of a defect. However, the process was laborious, time consuming, and therefore not readily translated to a clinical environment. Secondly, this treatment methodology required the engineered tissue to vascularize from the surrounding tissue. In patients with compromised vasculature, success rates could be potentially limited due to lack of oxygen and nutrient transport [45].

Researchers performed significant in vitro analysis of human BMSC interactions with various biomaterials. After selecting calcium phosphate cement (CPC) as a scaffolding material due to moldability, Weir and coworkers combined CPC, chitosan, and BMSCs to form a bone graft substitute [46]. While the primary purpose of this study was to investigate the mechanical properties of the composite, the BMSC differentiation toward an osteogenic pathway was evaluated. Primarily, the osteogenic potential of the BMSCs was investigated by quantifying alkaline phosphatase (ALP), an enzyme that marks osteogenic differentiation, and scaffold mineralization, which was detected by histological staining. By combining BMSCs with a CPC-chitosan composite, an in situ setting moldable scaffold with improved mechanical properties was created [46]. In this study, the group demonstrated the potential for use of primary cells that were harvested and placed onto three-dimensional scaffolds. However, this study was limited to in vitro experiments that do not demonstrate in vivo environmental conditions. For example, cell viability will be significantly altered when placed in a defect site with compromised vascularity.

Similarly, chitosan and biphasic calcium phosphate composites were evaluated for the in vivo regeneration of a $10-\mathrm{mm}$ transcortical mandibular defect [47]. While not a maxillary defect, correction of such a defect provides information that shares some similarities. In this study, porcine BMSCs were harvested from the femurs of pigs. Cells were expanded for a period of 2 weeks in osteogenic medium containing L-ascorbic acid 2-phosphate, $\beta$-glycerophosphate, and dexamethasone. BMSCs were combined with porous chitosan/biphasic calcium phosphate scaffolds with and without the presence of gelatin microspheres containing recombinant human basic human fibroblast growth factor (bFGF). The composites were then implanted into mandibular defects of the pigs from which the cells were retrieved. Throughout the study, the defects without bFGF did not completely regenerate. Therefore, incorporation of growth factors should be considered in reconstruction in the maxillofacial region. Of importance, calluses formed on the lateral side of the defect where periosteum was located, indicating the role of periosteum in fracture healing.

Mesenchymal stem cells have often also been used in sinus augmentation procedures. In order to maximize bone for dental implant placement, sinus augmentation is required to provide bone tissue for fixation. Typically, augmentation is performed through autologous grafts, but BMSCs have also been evaluated. In Iran, researchers successfully augmented significant bone mass for approximately $93 \%$ of 30 implants placed into several patients. In order to produce this amount of tissue, they combined hydroxyapatite and tricalcium phosphate with autologous BMSCs obtained from bone marrow aspirates. The cells were expanded in vitro and combined with the scaffold composite. The mucoperiosteal layer was pulled back and the composite with BMSCs was implanted into the defect site. Following implantation, a collagen membrane was placed between the mucoperiosteal layer and the composite to minimize soft tissue infiltration. The mucoperiosteal layer was then sutured back in place and the patient was allowed to heal for a period of 3 months. Following the conclusion of the study, patients had significant new bone growth, which allowed implants to be placed in the majority of the patients [48]. While this paper describes significant improvement in the alveolar bone thickness of the patient, acellular controls were not tested. Furthermore, the mucoperiosteal layer could potentially influence the outcome of the reconstruction.

Another study aimed to compare de novo bone formation from BMSCs with that realized by the "gold standard" of autologous cancellous bone. Sinus augmentation was performed on adult sheep. A comparison was made between autologous cancellous bone and BMSCs combined with bovine bone mineral. In this study, both groups facilitated similar bone growth. However, the control cancellous bone underwent a higher degree of resorption, thereby decreasing the volume of bone [49]. Furthermore, the amount of bone formation in scaffolds containing BMSCs was not related to the number of cells seeded. Therefore, evaluation of BMSCs must be performed and tailored to the individual patient.

BMSCs may be harvested from patients and concentrated, using two different methods involving synthetic polysaccharide and bone marrow aspirate concentrate methods. Cells were combined with bovine bone mineral to complete a sinus augmentation procedure on 18 adult human patients. As with the last study, a resorbable collagen matrix was used to protect the graft material from soft tissue infiltration. During the procedure, surgeons protected the mucoperiosteal flap and allowed the site to regenerate for a period of 3 months. Both concentration methods produced similar bone growth at the conclusion of the study [50]. For the purpose of the study, significant lamellar bone was created to allow dental implants. Overall, this study demonstrates the capability of BMSCs to 
help regenerate hard tissue. In this case, the surgery proved successful but required significant in vitro cell culture. Furthermore, the study did not evaluate bone regeneration in a complete defect model or an environment that was lacking adequate blood supply.

Using a rabbit model, researchers investigated the use of BMSCs combined with a porous ceramic scaffold to perform sinus augmentation procedures. Additionally, BMSCs were transduced with BMP-2 to evaluate the role of BMP-2 in sinus floor elevation in a rabbit model as compared with a negative control transduced with green fluorescent protein. Cells were harvested, then transduced using an adenovirus and expanded in vitro before being implanted on a porous ceramic scaffold. The composite was then implanted into the maxillary sinus of the rabbit for a period of 4 weeks. In this study, BMP-2 increased the amount of new bone area but did not significantly influence the bone height following sinus augmentation [51]. There are significant concerns regarding the use of genetic engineering when applied to in vivo treatments. In this study, however, the addition of genetic material did not appear to be necessary. Because the defect site was exposed, a possible solution was to apply an exogenous growth factor. However, this experimental model demonstrates the feasibility of improving bone regeneration through incorporation of growth factors.

Overall, BMSCs demonstrate an ability to differentiate into an osteogenic lineage and contribute to bone regeneration. While the use has been typically limited to a healthy patient model, the research illustrates the potential to be adapted to patients who have undergone treatment for oral cancer. In several cases, bone regeneration has been improved through the incorporation of growth factors such as bFGF and BMP-2, which could potentially be tailored for use in defect reconstruction $[47,51]$. Another potential application of BMSCs is their co-culture with endothelial cells, which has been shown to enhance the osteogenic differentiation of the BMSCs while simultaneously forming a three-dimensional prevascular network [52]. Furthermore, the care taken to protect the periosteum during a variety of procedures indicates the importance of such a tissue, which warrants further investigation $[47,50,51]$.

Periosteal-Derived Progenitor Cells Periosteum encases nearly every bone and contributes to normal physiology and regeneration (Fig. 3). The periosteum is divided into two layers, the outer layer and the cambium layer. The outer layer is mainly fibrous tissue, but also contains vasculature and a neural network that provides blood supply to the bone [53]. Due to this high level of vascularity, periosteum contains a significant number of endothelial cells and pericytes [54]. The cambium, or inner layer, contains PDPCs, fibroblasts, osteoblasts, and a microvascular network, which significantly impact bone homeostasis $[55,56]$. The PDPCs have been shown to be able to differentiate into osteogenic, adipogenic, chondrogenic, and myogenic lineages $[38,57,58]$.

Specifically, periosteum is responsible for bone healing following fracture. Callus formation is typically caused through the differentiation of PDPCs and production of a soft cartilage matrix, which is later remodeled. The disruption of the vasculature in the periosteum causes excess blood at the site. Periosteal wound healing follows the inflammation caused by blood loss. Similarly, vascular endothelial growth factor (VEGF) has been shown to stimulate angiogenesis for bone growth as well as signal osteoblast differentiation of progenitor cells $[59,60]$. Interactions between osteoprogenitor cells and endothelial cells are shown to simultaneously improve bone osteogenesis and vascular network formation $[52,61]$.

In addition to responding to specific growth factors, PDPCs have been shown to endogenously produce growth factors that can enhance functionality in a tissue-engineered construct. Specifically, BMP-2 has been shown to play a significant role in bone development in both ectopic and orthotopic bone growth $[62,63]$. PDPCs produce endogenous BMP-2, which serves in both paracrine and autocrine functionality $[64,65,66]$. PDPCs undergo chemotaxis toward cytokines such as thymus-expressed cytokine. This chemotactic ability provides the potential of implanting PDPCs near the defect and allowing migration to the defect site [67].

Periosteum thickness and functionalities vary with the following conditions: patient age and gender, the bone location and type $[53,54,68]$. The cambium layer of the periosteum is thickest during fetal development and begins to thin with age. The decrease in periosteal thickness is theorized to be a cause of increased osteoporosis [55]. In the case of elderly patients, the periosteum may be indistinguishable from the bone tissue [53].

Functionality of periosteum appears dependent on the type of bone from which the periosteum is harvested [69]. In a comparison of ectopic bone formation, tibial and calvarial periosteum were formed into a roll and implanted into the muscle of an adult rat. After 28 days, the tibial periosteum formed bone tissue through both endochondral and membranous ossification. However, the calvarial periosteum only formed bone through membranous ossification, which corresponds with fetal bone development [68]. Therefore, careful consideration of periosteal type should be made before using PDPCs in bone tissue engineering to ensure that bone type will be consistent with native tissue.

Expedited bone growth is essential for successful clinical reconstruction. Periosteal cells have demonstrated the capability to produce bone tissue quicker than BMSCs [70]. Furthermore, in vitro comparison of bone marrow-derived stem cells to periosteal-derived progenitor cells shows that PDPCs have higher osteogenic capability [71]. Histomorphometric analysis revealed periosteal cells 
Fig. 3 Description of periosteum by zone and cellular functionality [73]. Reprinted with permission from the International Journal of Dentistry

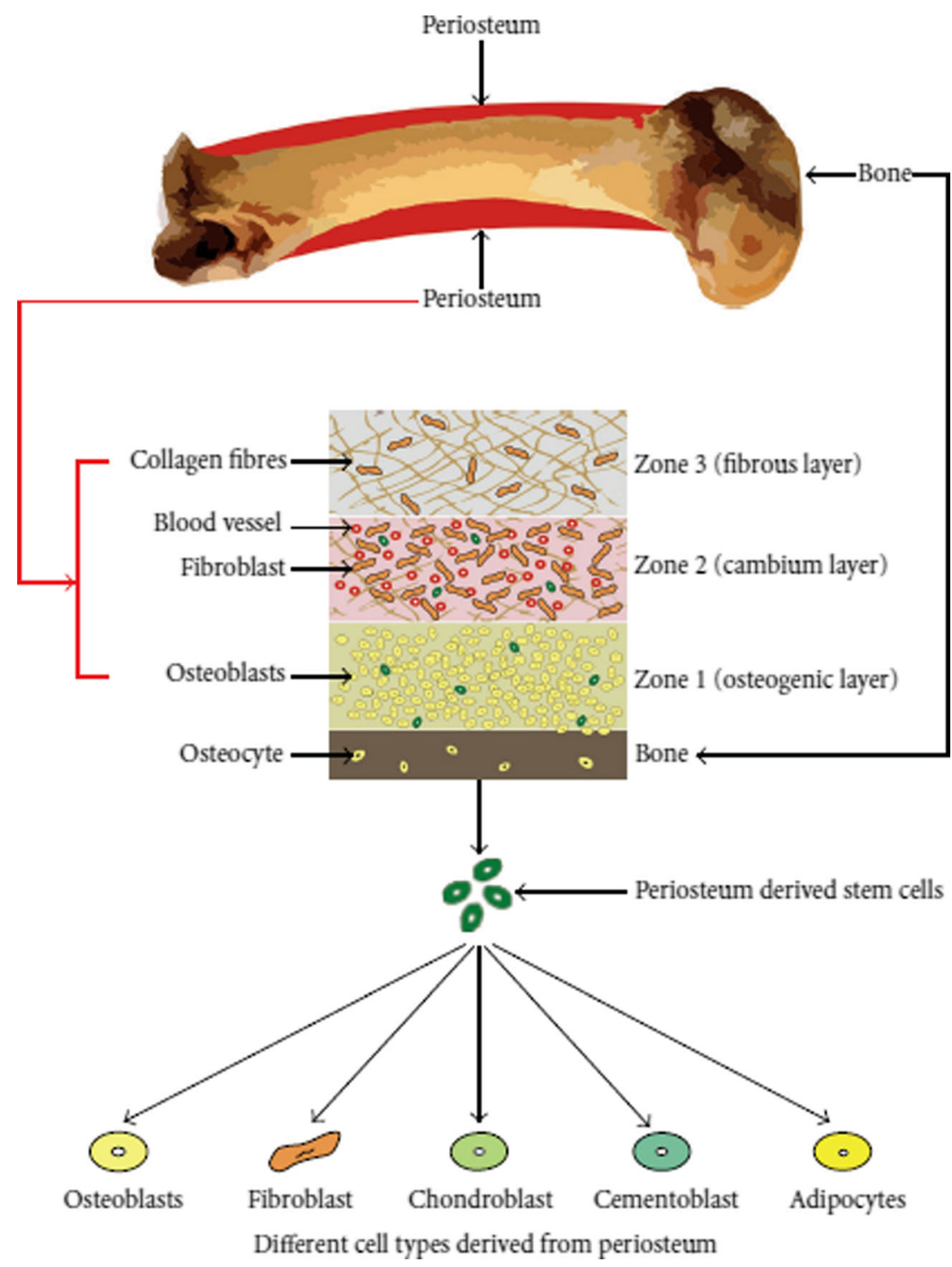

facilitated significantly more bone formation than BMSCs and alveolar bone cells, with new bone formation percentages of $58.2 \%$ (when derived with periosteal cells), $26.9 \%$ (when derived with BMSCs), and $41.1 \%$ (when derived with alveolar bone cells), respectively [72].

Periosteum Used as an Explant Traditionally, explants of periosteum have been used to promote osteogenesis. One of the most common uses of explanted periosteum is to correct periodontitis, which is a progressive degradation of the periodontal tissue, including alveolar bone. Research groups have recommended increased usage of periosteum to address issues with tissue degradation caused by diseases such as gingivitis [73]. In one study, by harvesting autologous periosteum and transplanting to the site of gingival recession defects, the periosteum showed excellent osteogenic potential and remodeled the defect site [74].
In a similar method to correct defects from periodontitis, a small explant of mandibular periosteum was biopsied from human patients to obtain a sample approximately $5 \times 5 \mathrm{~mm}^{2}$. The explant was then cultured in cell culture medium for approximately 30 days before being implanted and sewn into the defect site. For two of the patients, in this particular study, the defect site was too large for the periosteal graft. This issue was addressed by filling the defect site with platelet rich plasma (PRP). Following implantation, all patients had increases in alveolar bone at 4 months post-surgery [75]. In this study, the surgical procedure resulted in increased bone regeneration through periosteal explant transplantation, which facilitated improved mastication. However, the study required significant in vitro labor, which dramatically increased the expense of the procedure. Also, the role of the platelet rich plasma was not investigated. PRP has significant levels of autologous growth factors such as PDGF, TGF- $\beta$, and IGF-1, which have been shown to increase mitogenic activity of cells $[59,76,77,78]$. 
In addition to addressing periodontal issues, periosteal explants have been used in ectopic bone growth for transplantation to a critical size defect. In mature domestic sheep, periosteal strips were taken from the rib. The periosteal strips were placed on the top of chambers containing a bone graft, silk fibroin and chitosan scaffolds, or a blank control. Vascularity was assessed with a radiopaque dye. The animals were euthanized at days $7,14,21,41,63$, and 84 to evaluate the bone regeneration within the bone graft chamber. While analyzing samples using micro-CT for bone formation, the overall experiment demonstrates that periosteum is capable of forming ectopic bone growth in vivo. However, this procedure requires a total of four separate surgeries, which increase the odds of surgery related morbidity and mortality [79]. Furthermore, ectopic bone growth procedures would suffer from the same complications as free flap transfers; e.g., the amount of tissue might become too much for dental implantation. Additionally, these grafts lack epithelial layers that are required to recreate the soft tissue damage caused by the tumor resection.

\section{Periosteal-Derived Progenitor Cells Used in Tissue-} Engineered Constructs Despite the limited success of periosteum explants, the isolation of PDPCs has had some in vitro success. Following isolation of periosteal cells, flow cytometry can be performed to assess the presence of cell receptors commonly associated with PDPCs. For PDPCs obtained from human mandible, these receptors include CD9, CD105, and CD166, which are expressed through passage $15[38,80]$. Following isolation and passaging, differentiation medium can be used to differentiate PDPCs into adipogenic, osteogenic, and chondrogenic lineages. Assessment of protein expression is a common technique for evaluating differentiation. Specifically, osteocalcin and ALP expression are commonly evaluated to provide an assessment of osteogenic differentiation.

While investigating osteogenic and adipogenic potential of PDPCs harvested from human mastoid, Ringe and coworkers performed a detailed analysis of PDPCs and their osteogenic differentiation. Proliferation of the cells obtained from three separate donors was quantified. Variation in PDPCs proliferation with donor was observed. The proliferative capacity of the PDPCs suggests that there is potential for use of these cells in regenerative medicine applications. Following differentiation using various differentiation media, real-time polymerase chain reaction (RT-PCR) was used to investigate the expression of the following proteins: collagen type 1 alpha-1 (1A1), osteocalcin, adipocyte protein 2 (aP2), and clathrin-associated adaptor-protein-1 (APM1). Glyceraldehyde 3-phosphate dehydrogenase (GAPDH) was selected as the housekeeping gene. Following osteogenic differentiation, the cells demonstrated higher levels of osteocalcin, and APM1 [81]. Furthermore, von Kossa staining was performed on osteogenic media treated cells and demonstrated significant mineralization $[81,82]$. These in vitro experiments and others suggest that the potential for PDPCs in maxillary reconstruction is significant. Considerations must be made for periosteum harvest location as well as prolonged in vitro expansion, which may increase morbidity and expense, respectively.

In order to evaluate the functionality of differentiated PDPCs on a scaffold material, researchers have attempted to combine the cells with a variety of scaffold materials in hopes of finding a suitable material to repair critical size defects. In 1999, Redlich and coworkers added PDPCs, obtained from rabbit tibial periosteum, to polyglycolide-co-polylactide (PGL) fleece. The fleece scaffolds were cultured in osteogenic medium for several weeks. Cellular viability was assessed and immunohistochemistry evaluating ALP and osteocalcin expression was conducted. Polymer fleece seeded with PDPCs were implanted into rabbit calvarial defects. While the technique did not result in complete regeneration of the defect, the histological evaluation showed that this model demonstrated potential for PDPC use in critical size defects repairs [83]. The cells were differentiated prior to implantation, and demonstrated limited osteogenesis following implantation.

Due to the proliferative nature of the stem cells as well as the ability of PDPCs to express endogenous growth factors such as BMP-2, progenitor cells may have potential for implantation in a critical size defect $[65,66]$. With endogenous BMP-2 production, there is potential for enhanced osteogenesis by co-culturing PDPCs with BMSCs [84]. A $\beta$-tricalcium phosphate $(\beta-\mathrm{TCP})$ scaffold, seeded with both BMSCs and PDPCs, demonstrated enhanced ectopic bone growth and neovascularization in a nude mouse model.

Arnold and coworkers combined cells with PGL, but incorporated $\beta$-TCP. This procedure resulted in a "biocompatible" scaffold with osteoconductive and osteogenic properties. Using clinically available scaffolds from Ethicon, scaffolds were surface coated with thrombin and $\beta$ TCP was added. PDPCs were seeded on the surface and treated with osteogenic medium consisting of $10 \mathrm{mmol} / \mathrm{l} \beta$-glycerophosphate, $10^{-7} \mathrm{~mol} / 1$ dexamethasone, and $240 \mathrm{KIU} / \mathrm{ml}$ aprotinin for periods of $10,20,30$, and 40 days. Half of the constructs also received TGF- $\beta 1$ to increase osteogenesis. Assays were performed for deoxyribonucleic acid (DNA), osteocalcin, collagen, and ALP. Histological techniques were also performed. The constructs treated with TGF- $\beta 1$ had significantly more DNA, ALP, collagen, and osteocalcin than the constructs incubated in the absence of growth factors [85].

While investigating the cell-matrix interaction, the osteogenic potential of a fibrin, $\alpha$-tricalcium phosphate, and PDPC bead composite was evaluated. The beads were created by combining PDPCs with alginate, TCP, and fibrinogen. The solution was then dropped into a calcium chloride solution to produce beads. The alginate was then removed using a sodium citrate solution. Cell proliferation and differentiation were determined by cell count and detection of ALP activity and osteocalcin content. Cellular proliferation and osteogenic 
markers were elevated in beads incorporating TCP as compared with those that did not contain TCP [86]. A 3D bead culture system was previously established as a means to facilitate an injectable tissue-engineered construct and potentially reduce surgical complications as well as surgical cost [87]. However, the TCP study did not address in vivo reconstruction and the bead synthesis procedure outlined by this study is complicated and expensive [86].

In addition to fabrication of scaffold materials, Eyckmans and coworkers evaluated the use of commercially available bone graft substitutes. Following isolation of PDPCs from the tibia of rabbits, cells were expanded and frozen. Prior to implantation, cells were thawed and seeded onto two different commercially available scaffolds: Vitoss ${ }^{\mathrm{TM}}$ (65\% hydroxyapatite, $35 \%$ tricalcium phosphate and type I collagen) and Collagraft ${ }^{\mathrm{TM}}$ ( $\beta$-TCP and collagen). Approximately 20 million PDPCs were seeded onto each scaffold and each composite was placed into a titanium cup. The entire implant was then surgically placed into the skull of rabbits. After 12 weeks, the animals were euthanized and all implants revealed new bone formation. However, there was no significant difference between experimental and control groups, the latter containing scaffold material without cells. Specifically, the amount of orthotopic bone growth was not statistically significant [88]. In this study, the biomaterial composition had a marked impact on the type and amount of bone growth.

While the majority of research has incorporated a mineral component to regenerate bone tissue, Springer and coworkers performed in vivo sinus augmentation procedures in human patients to evaluate periosteum on a collagen matrix as compared with osteoblasts on demineralized bone matrix. All cells were harvested and cultured in vitro for a 1-week period before implantation. Bone matrix without cells was used as a control. Constructs that incorporated periosteal cells showed enough de novo bone density at 6 months to warrant dental implant placement; whereas, patients with other treatments required 8 months before dental implants could be placed [70]. While the study failed to compare different cells on the same biomaterial, the study indicates the potential for periosteal cells to repair a critical size defect in an expedited fashion as compared with osteoblasts.

Mechanical factors, in addition to biomaterial and media, can influence differentiation of PDPCs. While evaluating periosteum bone graft interaction, the following factors influence bone formation: mechanical loading, graft proximity to periosteum, and presence of a graft [89]. Other studies have shown that stretch of $1.5 \%$ applied to PDPCs causes an upregulation of TGF- $\beta$, which is an inducer of chondrogenic lineage $[88,90]$. In the past, bioreactors have been used to apply reproducible cyclic loads to osteoprogenitor cells [88, 91, 92].

\section{Biomaterials}

Ceramics Ceramic materials, such as $\beta$-tricalcium phosphate ( $\beta$-TCP), and hydroxyapatite (HA), are commonly used as bone graft substitutes due to high levels of biocompatibility, high compressive strength, and visualization using X-ray imaging. The ceramics are similar to that of the bone apatite found in bone, they allow cellular attachment and do not produce a large immune response [32, 93, 94]. In addition, ceramics are degradable by the body and can be remodeled during regeneration at different timeframes. These grafts can be manufactured into a variety of shapes and sizes, including injectable pastes, granules, strips, and disks [94, 95]. The manufacturing allows the pore size to be controlled, which is a significant factor in the resorption rate of the graft material [96]. For use as a bone graft, ceramics have demonstrated the ability to stimulate the process of bone regeneration within a critical size defect. A concern with ceramic bone grafts such as hydroxyapatite is the risk of possible infection, which is higher when the hydroxyapatite is in contact with the frontal sinus [97].

Commonly used for orthopedic surgeries, $\beta$-TCP grafts have been investigated for use as tissue engineering scaffold materials. TCP granules and strips have been evaluated for use in combination with numerous progenitor cell types such as mesenchymal stem cells, periosteal-derived progenitor cells, and adipose-derived stem cells $[85,98,99]$. The incorporation of a mineralized bone graft has been shown to promote cellular attachment, proliferation, the expression of osteogenic markers such as osteocalcin, type I collagen, and alkaline phosphatase $[85,86]$. Used in vivo, the combination of BMSCs and $\beta$-TCP has been highly successful in sinus augmentation procedures, allowing the majority of patients to have dental implants placed $[48,100]$.

While ceramics such as hydroxyapatite and $\beta$-TCP have demonstrated success as osteoconductive materials, tissueengineered constructs typically require prolonged growth and maturation prior to implantation. This process can increase the cost of the procedure and heavily relies on the ability of the patient's cells to infiltrate and vascularize the implant. One method of overcoming these limitations is to grow ectopic bone, and later transplant it to the defect site $[43,99,101]$. However, this approach increases the number of surgeries the patient must experience and increases the amount of time until the defect can be restored.

Natural Polymers Materials derived from biologic sources such as collagen, chitosan, and fibrin are commonly used in tissue engineering due to their ability to promote cellular attachment with minimal inflammation. Collagen has been used in craniofacial applications as membranes and bone graft fillers. For dental membrane applications, collagen grafts 
allow soft tissue infiltration, while maintaining the ability to be remodeled and resorbed [48]. For bone graft applications, biodegradable collagen sponges have been used in a variety of applications, including cranioplasty mandibular defect repairs and sinus augmentations. In sinus augmentations, collagen matrices combined with PDPCs regenerated sufficient bone for dental implantation after a period of 6 months [70]. Herford and Boyne showed similar results when applying a collagen matrix soaked in recombinant human bone morphogenetic protein-2 (rhBMP-2) to mandibular defects [102].

Chitosan is derived from the chitin found in the shells of crustaceans. Chitosan is a target for use as a tissue engineering scaffold in craniofacial applications due to its ability to be formed into microspheres, used as an injectable, or as a carrier agent for cells or growth factors [47, 103]. MSCs incorporated within microparticles have shown to be viable beyond 14 days [104]. Furthermore, the incorporation of calcium phosphates has been demonstrated to promote osteogenic differentiation and new bone growth. Osteoblasts are capable of migrating through chitosan pores and infiltrating composite materials [105].

Fibrin provides an interesting option as a tissue engineering scaffold. Fibrin is naturally occurring within the human body, can be remodeled easily, and can be polymerized in vitro. Similar to chitosan, fibrin can be used as a carrier agent to promote cellular viability and allow the incorporation of growth factors or ceramic compounds [86]. While fibrin itself does not promote osteogenic differentiation, fibrin scaffolds have been shown to increase cellular proliferation when used as cell carriers [40]. The incorporation of ceramic microparticles has been shown to promote osteogenic differentiation $[86,106]$.

Synthetic Polymers Polymeric scaffolds allow a great amount of variation and control during processing. Shapes ranging from lysosome nanoparticles to solid plates have been created and used in craniofacial applications [107, 108]. Typically, polylactide and polyglycolide are the most widely used polymers due to their mild inflammatory response, complete biodegradability, and Food and Drug Administrative approval for a variety of applications. However, polymeric biomaterials typically do not promote high levels of cellular attachment without either surface modification or the incorporation of a secondary material such as $\beta$-TCP, when compared with extracellular matrix based materials [109]. In vitro tissue engineering has shown poly-L-lactide-co-glycolide to be capable of allowing mineralization and osteogenic differentiation, as detected through histological and molecular characterization $[85,110,111]$. In vivo craniofacial applications of this approach have been varied, showing bone regeneration but demonstrated delayed healing in some cases [45, 83, 112].

\section{Growth Factors}

Frequently, tissue regeneration approaches incorporate exogenous growth factors such as basic fibroblast growth factor (bFGF), platelet derived growth factor (PDGF), and rhBMP-2 to enhance the bone regeneration constructs. Basic fibroblast growth factor and PDGF play roles as angiogenic, osteogenic, and mitogenic growth factors. The incorporation of growth factors into a tissue-engineered construct under specific experimental conditions has been shown to enhance vascularization, promote proliferation of the stem cells, and lead to an increase in in vitro osteogenic marker expression [47, 71, 77]. Similarly, the addition of bFGF increases the vascularization of newly formed ectopic bone [113].

In normal fracture healing, BMP-2 plays a significant role in the formation of mature osteoblasts and mineralized tissue [114]. The presence of rhBMP-2 has been shown to significantly enhance osteogenic differentiation and therefore has been widely used in a variety of tissue-engineered constructs [62]. However, a significant hurdle impeding the use of rhBMP-2 in tissue-engineered constructs is the cost. In lumbar fusions, the cost of rhBMP-2 can reach into the thousands per treatment [115]. In attempts to overcome this limitation, progenitor cells have been genetically modified, with success, to overexpress BMP-2 [43, 51].

Recently, the incorporation of platelet-rich plasma (PRP) has been investigated as an alternative to recombinant growth factors or genetic engineering. PRP is isolated by separating the platelets from the patient's own blood; it contains high levels of a variety of growth factors, including PDGF, TGF $\beta-1$, IGF-1, and VEGF [76, 78]. When combined with BMSCs, PRP has been shown to increased de novo bone formation in both sinus augmentation procedures as well as treatment of periodontal defects $[75,100]$.

\section{Discussion and Conclusion}

In the field of maxillofacial reconstruction, clinical treatments have room for significant improvement. Clinical treatments fail to restore optimal functional outcomes to patients who have undergone treatment for oral cancer, which leads to a decrease in physical and mental well-being. From the literature, it is clear that each treatment has beneficial qualities, but the treatments require optimization to produce a treatment that primarily restores functional outcomes, while simultaneously reducing the recovery time and the number of surgical procedures.

Throughout treatment, the functional outcomes of the patient are compromised. Of particular importance, mastication, swallowing, breathing, speaking, and ability to perform recreational and occupational activities are significantly diminished 
following oral cancer treatment $[5,16,17,21,23,116]$. Beyond the physical effects, these functional limitations often leave patients, who have already fought cancer, depressed and feeling helpless. In order to truly treat a patient, one must address more than the physical factors, i.e., one must also address the mental and emotional aspects of the disease. Continuing research in the field of maxillofacial reconstruction must aim to provide patients with restoration of functional outcomes and aesthetics in order to provide the patients with the highest possible quality of life.

Ideally, maxillofacial reconstruction involves complete regeneration of the host tissue to restore complete functional outcomes and aesthetics. While regenerative engineering provides the most potential to provide an ideal maxillofacial reconstruction, there are significant hurdles to overcome. First, tissue-engineered constructs require harvest and isolation of autologous cells, which requires additional surgical sites. Furthermore, the majority of research presented within this literature review involves in vitro characterization and cell expansion, which is laborious and expensive [44]. In an economic downturn, reconstructive approaches such as this may become prohibitively expensive. One solution is the possible isolation of localized tissue such as periosteum that can be removed with minimal complication at the time of the cancer treatment.

In order to optimize tissue-engineered constructs for maxillofacial reconstruction, procedures must be individually refined based on the type of biomaterial, cell type used, and the patient. Unfortunately, prolonged in vitro cell expansion and processing is prohibitively expensive and therefore other options must be evaluated. Maxillofacial reconstruction should begin to center about immediate transplantation of cells rather than prolonged in vitro cell culture, which reduces cost and reconstruction time. Due to the relatively new interest in this field of research, the literature currently does not reach consensus regarding biomaterials that would be applicable to the clinic [83]. In some cases, the mineral component of bone is required for bone tissue regeneration; whereas, other cases demonstrate de novo bone growth without bone mineral contained within the scaffold [70]. Therefore, biomaterial selection and characterization is critical to the success of maxillofacial reconstruction. Additionally, cell seeding efficiency, cell type, and incorporation of growth factors are variables that can be altered to expedite tissue regeneration $[101,116]$. The number of cells required to facilitate de novo bone formation has ranged widely in the literature, with one study indicating that more than 50 , 000 human PDPCs $/ \mathrm{mm}^{3}$ are required before the creation of new bone tissue [64].

Ultimately, the most important aspect of maxillofacial reconstruction must be the complete restoration of functionality so that patients can speak to their loved ones, return to a full diet, and breathe easily while simultaneously minimizing psychological decline. Unfortunately, none of the clinical treatments reliably restore this functionality. Therefore, a tissue-engineered construct capitalizing on the regeneration of the defect with healthy tissue warrants significant investigation due to its ability to provide restoration of lost tissue. Through optimization of the processing and scaffold material, the potential exists for expedited bone regeneration, minimized recovery time and medical costs. Overall, there is significant need for increased research in the area of regenerative engineering for maxillofacial reconstruction.

Specific areas of maxillofacial regenerative engineering need improvement. One area includes the development of a tissue-engineered maxillofacial construct that restores functional outcomes, while minimizing multiple surgeries and prolonged in vitro cellular expansion. Methodology to reproducibly create in vitro bone tissue must be created through optimization of culture media and growth factor incorporation. Potential progenitor cell source(s) require careful selection and characterization. The potential interaction between progenitor cells and native cells must be carefully analyzed. Scaffold materials must be selected and cell-material interaction must be evaluated. Finally, an adequate bench-top culture model [117] must be determined in order to recreate the necessary microenvironmental features of the oral cancer defect.

\section{References}

1. Shih A, Miaskowski C, Dodd MJ, Stotts NA, MacPhail L. Mechanisms for radiation-induced oral mucositis and the consequences. Cancer Nurs. 2003;26:222-9.

2. Siegel R, Naishadham D, Jemal A. Cancer statistics, 2012. CA Cancer J Clin. 2012;62:10-29.

3. Warnakulasuriya S. Living with oral cancer: epidemiology with particular reference to prevalence and life-style changes that influence survival. Oral Oncol. 2010;46:407-10.

4. Ram H, Sarkar J, Kumar H, Konwar R, Bhatt MLB, Mohammad S. Oral cancer: risk factors and molecular pathogenesis. J Oral Maxillofac Surg. 2011;10:132-7.

5. Marur S, D'Souza G, Westra WH, Forastiere AA. HPV-associated head and neck cancer: a virus-related cancer epidemic. Lancet. 2010;11:781-9.

6. Day TA, Davis BK, Gillespie MB, Joe JK, Kibbey M, Martin-Harris B et al. Oral cancer treatment. Curr Treat Opt Oncol. 2003;4:27-41.

7. Auluck A, Hislop G, Bajdik C, Poh C, Zhang L, Rosin M. Trends in oropharyngeal and oral cavity cancer incidence of human papillomavirus (HPV)-related and HPV-unrelated sites in a multicultural population: the British Columbia experience. Cancer. 2010;116:2635-44.

8. Chaturvedi AK, Engels EA, Anderson WF, Gillison ML. Incidence trends for human papillomavirus-related and unrelated oral squamous cell carcinomas in the United States. J Clin Oncol. 2008;26:612-9.

9. Chaturvedi AK, Engels EA, Pfeiffer RM. Human papillomavirus and rising oropharyngeal cancer incidence in the United States. J Clin Oncol. 2011;29:4294-301. 
10. Irish J, Sandhu N, Simpson C, Wood R, Gilbert R, Gullane P et al. Quality of life in patients with maxillectomy prostheses. Quality. 2009;813-21.

11. Zuydam AC, Lowe D, Brown JS, Vaughan ED, Rogers SN. Predictors of speech and swallowing function following primary surgery for oral and oropharyngeal cancer. Clin Otolaryngol. 2005;30:428-37.

12. Rogers SN, Lowe D, Fisher SE, Brown JS, Vaughan ED. Healthrelated quality of life and clinical function after primary surgery for oral cancer. Br J Oral Maxillofac Surg. 2002;40:11-8.

13. Shenoy VK, Shenoy K, Rodrigues S, Shetty P, Shenoy V, Article R. Management of oral health in patients irradiated for head and neck cancer: a review. Kathmandu Univ Med J. 2007;5:117-20.

14. Paris M, Chaux-Bodard A-G, Gourmet R, Fortin T. Guided implant surgery on oral cancer patients: in vitro study. Int J Comput Assist Radiol Surg. 2011;6:135-42.

15. Meijer GJ, de Bruijn JD, Koole R, van Blitterswijk CA. Cellbased bone tissue engineering. PLoS Med. 2007;4:e9.

16. Boyer AL, Butler EB, DiPetrillo TA, Engler MJ, Fraass B, Grant $\mathrm{W}$ et al. Intensity-modulated radiotherapy: current status and issues of interest. Int J Radiat Oncol Biol Phys. 2014;51:880-914.

17. Bucci MK, Bevan A, Roach M. Advances in radiation therapy: conventional to $3 \mathrm{D}$, to IMRT, to $4 \mathrm{D}$, and beyond. CA Cancer J Clin. 2005;55:117-34.

18. Huang S, Sullivan OB. Oral cancer: current role of radiotherapy and chemotherapy. Med Oral Patol Oral y Cir Bucal. 2013;18: e233-40.

19. Trotti A. Toxicity in head and neck cancer: a review of trends and issues. Int J Radiat Oncol Biol. 2000;47:1-12.

20. Javed F, Al-Hezaimi K, Al-Rasheed A, Almas K, Romanos GE. Implant survival rate after oral cancer therapy: a review. Oral Oncol. 2010;46:854-9.

21. Ihde S, Kopp S, Gundlach K, Konstantinović VS. Effects of radiation therapy on craniofacial and dental implants: a review of the literature. Oral Surg Oral Med Oral Pathol Oral Radiol Endod. 2009;107:56-65.

22. Mahanna GK, Gaebler CA, Sullivan M. Obturator prostheses after cancer surgery: an approach to speech outcome assessment. J Prosthet Dent. 1998;79:310-6.

23. Lethaus B, Lie N, de Beer F, Kessler P, de Baat C, Verdonck HW. Surgical and prosthetic reconsiderations in patients with maxillectomy. J Oral Rehabil. 2010;37:138-42.

24. Scully C, Porter S. Oral cancer. West J Med. 2001;174:348-51.

25. Peng X, Mao C, Yu G, Guo C, Huang M, Zhang Y. Maxillary reconstruction with the free fibula flap. Plast Reconstr Surg. 2005;115:1562-9.

26. Santamaria E, Cordeiro PG. Reconstruction of maxillectomy and midfacial defects with free tissue transfer. J Surg Oncol. 2006;94: $522-31$

27. Bianchi B, Ferri A, Ferrari S, Copelli C, Sesenna E. Maxillary reconstruction using anterolateral thigh flap and bone grafts. Microsurgery. 2009. doi:10.1002/micr.

28. Epstein JB, Emerton S, Kolbinson DA, Le ND, Phillips N, Stevenson-Moore $\mathrm{P}$, et al. Quality of life and oral function following radiotherapy for head and neck cancer. Head Neck. 1999;21: $1-11$

29. Hassanein KA-AM, Musgrove BT, Bradbury E. Psychological outcome of patients following treatment of oral cancer and its relation with functional status and coping mechanisms. J Craniomaxillofac Surg. 2005;33:404-9.

30. Genden EM, Okay D, Stepp MT, Rezaee RP, Mojica JS, Buchbinder D, et al. Comparison of functional and quality-oflife outcomes in patients with and without palatomaxillary reconstruction: a preliminary report. Arch Otolaryngol Head Neck Surg. 2003;129:775-80.
31. Bianco P, Riminucci M, Gronthos S, Robey PG. Bone marrow stromal stem cells: nature, biology, and potential applications. Stem Cells. 2001;19:180-92.

32. Mastrogiacomo M, Muraglia A, Komlev V, Peyrin F, Rustichelli F, Crovace A, et al. Tissue engineering of bone: search for a better scaffold. Orthod Craniofac Res. 2005;8:277-84.

33. Majumdar MK, Thiede MA, Mosca JD, Moorman M, Gerson SL. Phenotypic and functional comparison of cultures of marrowderived mesenchymal stem cells (MSCs) and stromal cells. J Cell Physiol. 1998;176:57-66.

34. Olivier V, Faucheux N, Hardouin P. Biomaterial challenges and approaches to stem cell use in bone reconstructive surgery. Drug Discov Today. 2004;9:803-11.

35. Mao JJ, Giannobile WV, Helms JA, Hollister SJ, Krebsbach PH, Longaker MT, et al. Craniofacial tissue engineering by stem cells. J Dent Res. 2006;85:966-79.

36. Jones EA, Kinsey SE, English A, Jones RA, Straszynski L, Meredith DM, et al. Isolation and characterization of bone marrow multipotential mesenchymal progenitor cells. Arthritis Rheum. 2002;46:3349-60.

37. Zhou J, Shi S, Shi Y, Xie H, Chen L, He Y, et al. Role of bone marrow-derived progenitor cells in the maintenance and regeneration of dental mesenchymal tissues. J Cell Physiol. 2011;226: 2081-90.

38. Park B-W, Hah Y-S, Kim DR, Kim J-R, Byun J-H. Osteogenic phenotypes and mineralization of cultured human periostealderived cells. Arch Oral Biol. 2007;52:983-9.

39. Belmar-Lopez C, Mendoza G, Oberg D, Burnet J, Simon C, Cervello I, et al. Tissue-derived mesenchymal stromal cells used as vehicles for anti-tumor therapy exert different in vivo effects on migration capacity and tumor growth. BMC Med. 2013;11:139.

40. Demol J, Eyckmans J, Roberts SJ, Luyten FP, Van Oosterwyck H Does tranexamic acid stabilised fibrin support the osteogenic differentiation of human periosteum derived cells? Eur Cell Mater. 2011;21:272-85.

41. Spaeth E, Klopp A, Dembinski J, Andreeff M, Marini F. Inflammation and tumor microenvironments: defining the migratory itinerary of mesenchymal stem cells. Gene Ther. 2008;15: 730-38.

42. Spaeth EL, Kidd S, Marini FC. Tracking inflammation-induced mobilization of mesenchymal stem cells. Methods Mol Biol. 2012;904:173-90.

43. Steinhardt Y, Aslan H, Regev E, Zilberman Y, Kallai I, Gazit D, et al. Maxillofacial-derived stem cells regenerate critical mandibular bone defect. Tissue Eng Part A. 2008;14:1763-73.

44. Lucaciu O, Băciuț M, Băciuț G, Câmpian R, Sorițău O, Bran S, et al. Tissue engineered bone versus alloplastic commercial biomaterials in craniofacial reconstruction. Rom J Morphol Embryol. 2010;51:129-36.

45. Schimming R. Tissue-engineered bone for maxillary sinus augmentation. J Oral Maxillofac Surg. 2004;62:724-9.

46. Weir MD, Xu HHK. Culture human mesenchymal stem cells with calcium phosphate cement scaffolds for bone repair. J Biomed Mater Res Part B Appl Biomater. 2010;93:93-105.

47. Urkmez AS, Clark SG, Wheeler MB, Goldwasser MS, Jamison RD. Evaluation of chitosan/biphasic calcium phosphate scaffolds for maxillofacial bone tissue engineering. Macromol Symp. 2008;269:100-5

48. Shayesteh YS, Khojasteh A, Soleimani M, Alikhasi M, Khoshzaban A, Ahmadbeigi N. Sinus augmentation using human mesenchymal stem cells loaded into a beta-tricalcium phosphate/ hydroxyapatite scaffold. Oral Surg Oral Med Oral Pathol Oral Radiol Endod. 2008;106:203-9.

49. Gutwald R, Haberstroh J, Kuschnierz J, Kister C, Lysek DA, Maglione M, et al. Mesenchymal stem cells and inorganic bovine bone mineral in sinus augmentation: comparison with 
augmentation by autologous bone in adult sheep. Br J Oral Maxillofac Surg. 2010;48:285-90.

50. Sauerbier S, Stricker A, Kuschnierz J, Bühler F, Oshima T, Xavier $\mathrm{SP}$, et al. In vivo comparison of hard tissue regeneration with human mesenchymal stem cells processed with either the FICOLL method or the BMAC method. Tissue Eng Part C Methods. 2010;16:215-23.

51. Sun X-J, Xia L-G, Chou LL, Zhong W, Zhang X-L, Wang S-Y, et al. Maxillary sinus floor elevation using a tissue engineered bone complex with BMP-2 gene modified bMSCs and a novel porous ceramic scaffold in rabbits. Arch Oral Biol. 2010;55:195-202.

52. Rouwkema J, de Boer J, Blitterswijk VCA. Endothelial cells assemble into a 3-dimensional prevascular network in a bone tissue engineering construct. Tissue Eng. 2006;12:2685-93.

53. Dwek JR. The periosteum: what is it, where is it, and what mimics it in its absence? Skelet Radiol. 2010;39:319-23.

54. Allen MR, Hock JM, Burr DB. Periosteum: biology, regulation, and response to osteoporosis therapies. Bone. 2004;35:1003-12.

55. Arnsdorf EJ, Jones LM, Carter DR, Jacobs CR. The periosteum as a cellular source for functional tissue engineering. Tissue Eng Part A. $2009 ; 15: 2637-42$.

56. Squier CA, Ghoneim S, Kremenak CR. Ultrastructure of the periosteum from membrane bone. J Anat. 1990;171:233-9.

57. De Bari C, Dell'Accio F, Luyten FP. Human periosteum-derived cells maintain phenotypic stability and chondrogenic potential throughout expansion regardless of donor age. Arthritis Rheum. 2001;44:85-95.

58. De Bari C, Dell'Accio F, Vanlauwe J, Eyckmans J, Khan IM, Archer CW, et al. Mesenchymal multipotency of adult human periosteal cells demonstrated by single-cell lineage analysis. Arthritis Rheum. 2006;54:1209-21.

59. Malizos KN, Papatheodorou LK. The healing potential of the periosteum molecular aspects. Injury. 2005;36 Suppl 3:S13-9.

60. Schindeler A, McDonald MM, Bokko P, Little DG. Bone remodeling during fracture repair: the cellular picture. Semin Cell Dev Biol. 2008;19:459-66.

61. Moioli EK, Clark PA, Xin X, Lal S, Mao JJ. Matrices and scaffolds for drug delivery in dental, oral and craniofacial tissue engineering. Adv Drug Deliv Rev. 2007;59:308-24.

62. Cheng H, Jiang W, Phillips FM, Haydon RC, Peng Y, Zhou L, et al. Osteogenic activity of the fourteen types of human bone morphogenetic proteins (BMPs). J Bone Joint Surg Am. 2003;85-A: 1544-52.

63. Kang Q, Sun MH, Cheng H, Peng Y, Montag AG, Deyrup AT, et al. Characterization of the distinct orthotopic bone-forming activity of 14 B.P. using recombinant adenovirus-mediated gene delivery. Gene Ther. 2004;11:1312-20.

64. Eyckmans J, Roberts SJ, Schrooten J, Luyten FP. A clinically relevant model of osteoinduction: a process requiring calcium phosphate and BMP/Wnt signalling. J Cell Mol Med. 2010;14: 1845-56.

65. Ueno T, Kagawa T, Kanou M, Fujii T, Fukunaga J. Immunohistochemical observations of cellular differentiation and proliferation in endochondral bone formation from grafted periosteum: expression and localization of BMP-2 and -4 in the grafted periosteum. J Craniomaxillofac Surg. 2003;5182:356-61.

66. Wang Q, Huang C, Xue M, Zhang X. Expression of endogenous BMP-2 in periosteal progenitor cells is essential for bone healing. Bone. 2011;48:524-32.

67. Stich S, Loch A, Leinhase I, Neumann K, Kaps C, Sittinger M, et al. Human periosteum-derived progenitor cells express distinct chemokine receptors and migrate upon stimulation with CCL2, CCL25, CXCL8, CXCL12, and CXCL13. Eur J Cell Biol. 2008;87:365-76
68. Fujii T, Ueno T, Kagawa T, Sakata Y, Sugahara T. Comparison of bone formation ingrafted periosteum harvested from tibia and calvaria. Microsc Res Tech. 2006;69(7):580-4.

69. Allen MR, Burr DB. Human femoral neck has less cellular periosteum, and more mineralized periosteum, than femoral diaphyseal bone. Bone. 2005;36:311-6.

70. Springer IN, Nocini PF, Schlegel KA, De Santis D, Park J, Warnke $\mathrm{PH}$, et al. Two techniques for the preparation of cell-scaffold constructs suitable for sinus augmentation: steps into clinical application. Tissue Eng. 2006;12:2649-56.

71. Agata H, Asahina I, Yamazaki Y, Uchida M, Shinohara Y, Honda MJ, et al. Effective bone engineering with periosteum-derived cells. J Dent Res. 2007;86:79-83.

72. Zhu S-J, Choi B-H, Huh J-Y, Jung J-H, Kim B-Y, Lee S-H. A comparative qualitative histological analysis of tissue-engineered bone using bone marrow mesenchymal stem cells, alveolar bone cells, and periosteal cells. Oral Surg Oral Med Oral Pathol Oral Radiol Endod. 2006;101:164-9.

73. Mahajan A. Periosteum: a highly underrated tool in dentistry. Int J Dent. 2012;717816.

74. Caria PH, Kawachi EY, Bertran CA, Camilli JA. Biological assessment of porous-implant hydroxyapatite combined with periosteal grafting in maxillary defects. J Oral Maxillofac Surg. 2007;65:847-54.

75. Mizuno H, Kagami H, Mase J, Mizuno D, Ueda M. Efficacy of membranous cultured periosteum for the treatment of patients with severe periodontitis: a proof-of-concept study. Najoya Med J. 2010;72:59-70.

76. Weibrich G, Kleis WKG, Hafner G, Hitzler WE. Growth factor levels in platelet-rich plasma and correlations with donor age, sex, and platelet count. J Craniomaxillofac Surg. 2002;30:97-102.

77. Vavouraki H, Dereka X, Vrotsos I, Markopoulou C. Ability of a bovine bone graft, alone or enriched with PDGF-BB or rhBMP-2, to promote human periodontal ligament (PDL) cells proliferation. a preliminary study. Cell Tissue Bank. 2003;4:17-23.

78. Schmidmaier G, Herrmann S, Green J, Weber T, Scharfenberger A, Haas NP, et al. Quantitative assessment of growth factors in reaming aspirate, iliac crest, and platelet preparation. Bone. 2006;39:1156-63.

79. Ríos CN, Skoracki RJ, Miller MJ, Satterfield WC, Mathur AB. In vivo bone formation in silk fibroin and chitosan blend scaffolds via ectopically grafted periosteum as a cell source: a pilot study. Tissue Eng Part A. 2009;15:2717-25.

80. Choi Y-S, Noh S-E, Lim S-M, Lee C-W, Kim C-S, Im M-W, et al. Multipotency and growth characteristic of periosteum-derived progenitor cells for chondrogenic, osteogenic, and adipogenic differentiation. Biotechnol Lett. 2008;30:593-601.

81. Ringe J, Leinhase I, Stich S, Loch A, Neumann K, Haisch A. Human mastoid periosteum-derived stem cells: promising candidates for skeletal tissue engineering. Tissue Eng. 2008;2:136-46.

82. Hutmacher DW, Sittinger M. Periosteal cells in bone tissue engineering. Tissue Eng. 2003;9 Suppl 1:S45-64.

83. Redlich A, Perka C, Schultz O, Spitzer R, Häupl T, Burmester GR, et al. Bone engineering on the basis of periosteal cells cultured in polymer fleeces. J Mater Sci Mater Med. 1999;10:767-72.

84. Jiang Y, Shen H, He Y, Lu J, Chen D, Zhang X, et al. Co-culturing mesenchymal stem cells from bone marrow and from periosteum accelerates osteogenesis and neovascularization for bone tissue engineering. J Tissue Eng Regen Med. 2010;6:822-32.

85. Arnold U, Lindenhayn K, Perka C. In vitro-cultivation of human periosteum derived cells in bioresorbable polymer-TCP-composites. Biomaterials. 2002;23:2303-10.

86. Spitzer R, Perka C, Lindenhayn K, Zippel H. Matrix engineering for osteogenic differentiation of rabbit periosteal cells using $\alpha$ tricalcium phosphate particles in a three-dimensional fibrin culture. Vitr Cell Dev Biol Anim. 2001;59:690-6. 
87. Burg KJL, Boland T. Bioengineered devices: minimally invasive tissue engineering composites and cell printing. IEEE EMB. 2003;22:84-91.

88. Eyckmans J (2007) Periosteum derived progenitor cells in bone tissue engineering. Katholieke Universiteit Leuven.

89. Knothe UR, Dolejs S, Matthew Miller R, Knothe Tate ML. Effects of mechanical loading patterns, bone graft, and proximity to periosteum on bone defect healing. J Biomech. 2010;43:2728-37.

90. Kock LM, Ravetto A, van Donkelaar CC, Foolen J, Emans PJ, Ito $\mathrm{K}$. Tuning the differentiation of periosteum-derived cartilage using biochemical and mechanical stimulations. Osteoarthr Cartil. 2010;18:1528-35.

91. Orr DE, Burg KJL. Design of a modular bioreactor to incorporate both perfusion flow and hydrostatic compression for tissue engineering applications. Ann Biomed Eng. 2008;36:1228-41.

92. Depprich R, Handschel J, Wiesmann H-P, Jäsche-Meyer J, Meyer U. Use of bioreactors in maxillofacial tissue engineering. Br J Oral Maxillofac Surg. 2008;46:349-54.

93. Ogose A, Hotta T, Hatano H, Kawashima H, Tokunaga K, Endo $\mathrm{N}$, et al. Histological examination of beta-tricalcium phosphate graft in human femur. J Biomed Mater Res. 2002;63:601-4.

94. Daculsi G. Biphasic calcium phosphate concept applied to artificial bone, implant coating and injectable bone substitute. Biomaterials. 1998;19:1473-8.

95. Bone C, Chan WD, Perinpanayagam H, Goldberg HA, Hunter GK, Dixon SJ, et al. Tissue engineering scaffolds for the regeneration of craniofacial bone. J Can Dent Assoc. 2009;75:373-7.

96. von Doernberg M-C, von Rechenberg B, Bohner M, Grünenfelder $\mathrm{S}$, van Lenthe GH, Müller R, et al. In vivo behavior of calcium phosphate scaffolds with four different pore sizes. Biomaterials. 2006;27:5186-98.

97. Verret DJ, Ducic Y, Oxford L, Smith J. Hydroxyapatite cement in craniofacial reconstruction. Otolaryngol - Head Neck Surg. 2005;133:897-9.

98. Brown RQ, Mount A, Burg KJL. Evaluation of polymer scaffolds to be used in a composite injectable system for intervertebral disc tissue engineering. J Biomed Mater Res A. 2005;74:32-9.

99. Mesimäki K, Lindroos B, Törnwall J, Mauno J, Lindqvist C, Kontio R, et al. Novel maxillary reconstruction with ectopic bone formation by GMP adipose stem cells. Int J Oral Maxillofac Surg. 2009;38:201-9.

100. Yamada Y, Nakamura S, Ito K, Kohgo T, Hibi H, Nagasaka T, et al. Injectable tissue-engineered bone using autogenous bone marrow-derived stromal cells for maxillary sinus augmentation: clinical application report from a 2-6-year follow-up. Tissue Eng Part A. 2008;14:1699-707.

101. Eyckmans J, Luyten FP. Species specificity of ectopic bone formation using periosteum-derived mesenchymal progenitor cells. Tissue Eng. 2006;12:2203-13.

102. Herford AS, Boyne PJ. Reconstruction of mandibular continuity defects with bone morphogenetic protein-2 (rhBMP-2). J Oral Maxillofac Surg. 2008;66:616-24.
103. Di Martino A, Sittinger M, Risbud MV, Di A. Chitosan: a versatile biopolymer for orthopaedic tissue-engineering. Biomater. 2005;26:5983-90.

104. García Cruz DM, Escobar Ivirico JL, Gomes MM, Gómez Ribelles JL, Sánchez MS, Reis RL, et al. Chitosan microparticles as injectable scaffolds for tissue engineering. JTERM. 2008;2: 378-80.

105. $\mathrm{Xu} \mathrm{HH,} \mathrm{Weir} \mathrm{MD,} \mathrm{Simon} \mathrm{Jr} \mathrm{CG.} \mathrm{Injectable} \mathrm{and} \mathrm{strong} \mathrm{nano-}$ apatite scaffolds for cell/growth factor delivery and bone regeneration. Dent Mater. 2008;24:1212-22.

106. Yamada Y. Bone regeneration following injection of mesenchymal stem cells and fibrin glue with a biodegradable scaffold. J CranioMaxillofacial Surg. 2003;31:27-33.

107. Joo V, Ramasamy T, Haidar ZS. A novel self-assembled liposomebased polymeric hydrogel for cranio-maxillofacial applications: preliminary findings. Polymers. 2011;14:967-74.

108. Suuronen R, Pohjonen T, Hietanen J, Lindqvist C. A 5-year in vitro and in vivo study of the biodegradation of polylactide plates. J Oral Maxillofac Surg. 1998;56:604-14.

109. Abayasinghe NK, Perera KP, Thomas C, Daly A, Suresh S, Burg $\mathrm{K}$, et al. Amido-modified polylactide for potential tissue engineering applications. J Biomater Sci Polym Ed. 2004;15:595-606.

110. Abukawa H, Terai H, Hannouche D, Vacanti JP, Kaban LB, Troulis MJ. Formation of a mandibular condyle in vitro by tissue engineering. J Oral Maxillofac Surg. 2003;61:94-100.

111. Sakata Y, Ueno T, Kagawa T, Kanou M, Fujii T, Yamachika E, et al. Osteogenic potential of cultured human periosteum-derived cells - a pilot study of human cell transplantation into a rat calvarial defect model. J Craniomaxillofac Surg. 2006;34:461-5.

112. Schantz JT, Hutmacher DW, Lam CXF, Brinkmann M, Wong KM, Lim TC, et al. Repair of calvarial defects with customised tissue engineered bone grafts II. Evaluation of cellular efficiency and efficacy in vivo. Tissue Eng. 2003;9:S127-39.

113. Wiltfang J, Merten HA. Ectopic bone formation with the help of growth factor bFGF. J Craniomaxillofac Surg. 1996;24:300-4.

114. Yang W, Guo D, Harris MA. BMP2 in osteoblasts of periosteum and trabecular bone links bone formation to vascularization and mesenchymal stem cells. J Cell Sci. 2013;126:4085-98.

115. Polly DW, Ackerman SJ, Shaffrey CI, Ogilvie JW, Wang JC, Stralka SW, et al. A cost analysis of bone morphogenetic protein versus autogenous iliac crest bone graft in single-level anterior lumbar fusion. Orthopedics. 2003;26:1027-37.

116. Impens S, Chen Y, Mullens S, Luyten F, Schrooten J. Controlled cell-seeding methodologies: a first step toward clinically relevant bone tissue engineering strategies. Tissue Eng Part $\mathrm{C}$ Methods. 2010;16:1575-83.

117. Burg TC, Parzel CA, Groff RE, Pepper M, Burg KJL. Building off-the-shelf tissue engineered composites. Phil Trans R Soc A. 2010;368:1839-62. 